\title{
ENFERMEDAD DE HAND SCHULLER CHRISTIAN
}

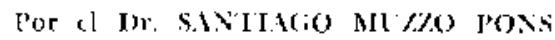

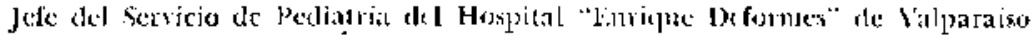

Muy pocos son los casos deseritos en la literatura mundial de la enfermedad de HandSchüller-Christian, alrededor de 100 solamente. En nuestro país, revisando las publicaciones pediátricas, encontramos que el primer caso fué presentado por el Profesor. Scroggie el año 1930 en su trabajo "Cráneo geográfico hipofisiario", con la observación de una niñita de 3 años de edad (Archivos del Hospital Roberto del Río). Junto con este caso publica todos los aparecidos en la literatura pediátrica mundial hasta ese tiempo, casos que totalizan 11 .

Posteriormente, Kohan, en 1933 relata otro caso presentado en un joven de 18 años y por fin, en 1940, Armas Cruz, Montero y Diaz publican un tercer caso ocurrido en una niñita de 9 años y 8 meses.

Fuera de los casos de marcha típica, hay un buen número de formas atípicas que han venido a aclarar muchos puntos de su patogenia, relacionando las diversas formas anátomo-clinicas de esta enfermedad.

El año 1944, el Profesor Baeza Goñi y el Dr. J. Espinoza Soto publican un caso de marcha atípica en un niño de 3 años 2 meses y hacen una revisión de todos los cuadros clínicos que genéricamente pueden englobarse dentro de la denominación de "reticulo endoteliosis".

Definición. La enfermedad de Hand-Schüller-Christian es probablemente un desorden heredo familiar constitucional deI metabolismo, en el cual se produce un depósito de lípidos en los tejidos, particularmente colesterol y sus ésteres, lo cual conduce a una reacción hiperplástica de las células retículo endoteliales (histiocitos).

Historia. El primer caso fué descrito por Hand el año 1893, en un niño de 3 años de edad. En 1915, Schüiler estudia las lesiones radiológicas en 2 casos más y en 1919 Christian hizo una admirable descripción clínica de la enfermedad, presentando el cuadro con sus tres sintomas dominantes: defectos de los huesos membranosos, exoftalmus $y$ dia- betes insípida; pero es Rowland, en 1928 , quien da la interpretación patogénica de este proceso como un trastorno primario en el metabolismo de los lípidos con almacenamiento de colesterol y sus ésteres y con una hiperplasia secundaria del sistema retículo endotelial.

Clasificación de las retículo endoteliosis lipidas. Según el concepto moderno basado en la similitud de caracteres químico-histoIógicos y clínico-evolutivos de las diversas xantomatosis lípidas, éstas se pueden reunir en dos subgrupos: el primero está centrado por la enfermedad de Hand-Schuiller-Christian, leniendo cono acompañantes a la enfermedad de Abbot-Letterer-Siwe $y$ al granuloma eosinófilo, ambas consideradas hoy como modalidades de un mismo proceso. El segundo subgrupo tiene como cuadro principal a la enfermedad de Gaucher con sus dos formas: aguda y crónica, incluyéndose hoy er su órbita la enfermedad de Niemann Pick y también la enfermedad de Tay Sachs, aunque en esta última la infiltración lípida sì hace en células de origen ectodérmico. Nos referiremos sólo a la enfermedad de Hand-Schüller-Christian, motivo de esta presentación.

Frecuencia, edad, raza y sexo. Es una enfermedad más común en los niños que en los adultos. Se ve en la segunda infancia $y$ es muy rara en los lactantes. No tiene predilección por determinadas razas, como la enfermedad de Niemann Pick. Los varones son más atacados que las niñas en proporción de 3 a 2 (Rowland). Parece haber tendencia familiar. La xantomatosis crónica ha sido descrita en todos los países del mundo: Europa, Australia, Japón y ambas Américas. Un tercio de los casos descritos han sido observados en Estados Unidos. La mayoría de los casos han sido descritos en los últimos 5 años y son del tipo de Hand-SchüllerChristian.

Estudio clínico. Los síntomas más característicos son los defectos en los huesos mem- 
branosos, exoftalmo $\mathrm{y}$ diabetes insf́pida. A esta tríada se agregan las lesiones xantomatosas de la piel. Pero estos síntomas pueden producirse separadamente o faltar, siendo la diabetes insipida la que falta con mayor frecuencia.

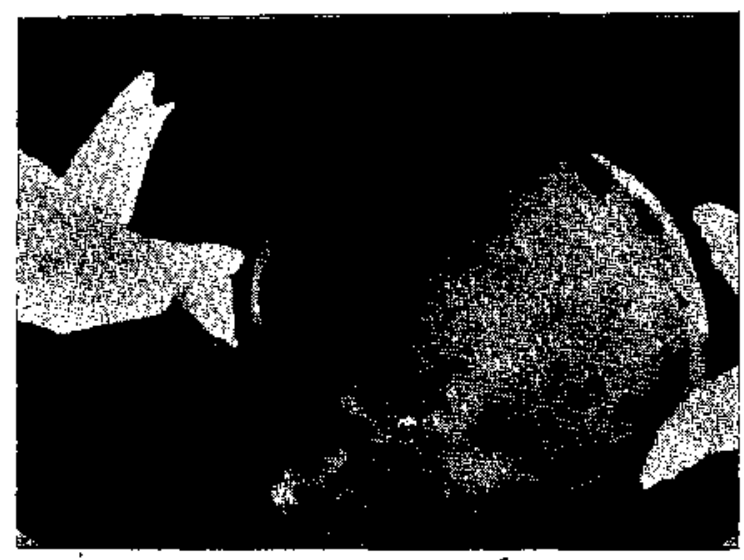

Gráfico No 1

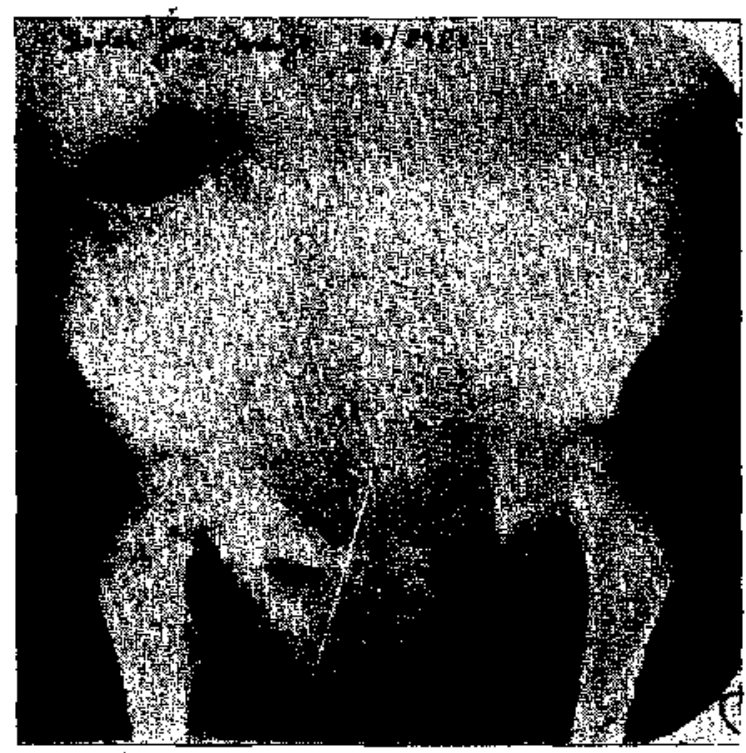

Gráfico No 2

Su comienzo es insidioso y se inicia, por Io general, antes de los 6 años de edad, pasando mucho tiempo confundido con otros procesos, hasta que aparece la tríada sintomática característica. No son raros los casos en que durante algún tiempo sólo aparece la lesión granulomatosa, como granulomas aislados y sólo mucho más tarde se identifica como enfermedad de Hand-Schüller-
Christian. Los sintomas más frecuentes son las lagunas óseas, siendo el cráneo la región esquelética más atacada (cráneo geográfico o en mapa). Estas lesiones se caracterizan por pérdida de sustancia y en algunos casos se inicia con condensación o marmorizaciones (Hofer). Completa estas lesiones la infiltración de masas xantomatosas. Las imágenes radiológicas del cráneo son tan particulares (figura 1), que habiéndolas visto una vez no se olvidan más. Otros huesos también pueden ser afectados (ilfaco, omoplatos, fémur, etc.) (figuras 2 y 3 ).

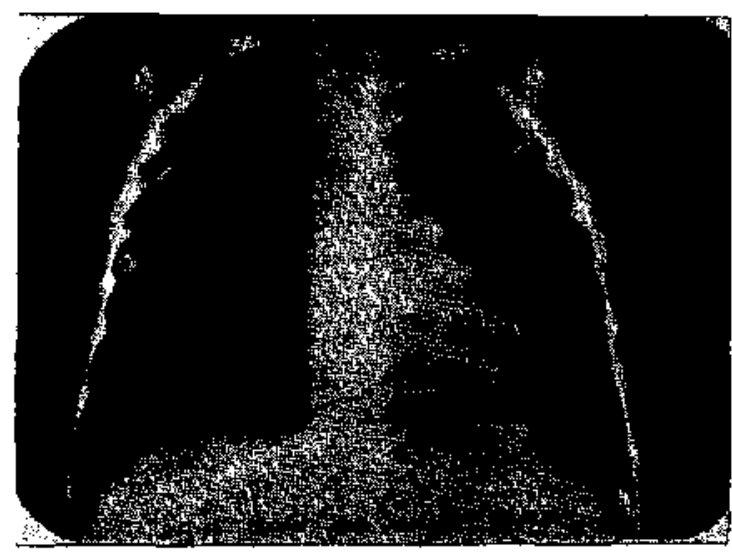

Gráfico No ?

La exoftalmia (figura 4) es uno de los síntomas más constantes y es muy pronunciada on los casos avanzados. Puede ser uni o bilateral, llegando a veces a la enucleación casi total por la acumulación de masas xantomatósicas en la región orbitaria. La diabetes insípida se debe a la repercusión de las lesiones óseas sobre el hipotálamo, en las vecindades de la hipófisis, pudiendo tam. bién presentarse por las mismas razones el sindroma adiposo genital y el infantilismo.

Otras lesiones. Pueden presentarse, adefás, xantomas cutáneos, asi como manchas amarillas, granulomas y necrosis de las encías con expulsión de dientes (enfermedad de Abbott-Letterer-Siwe).

En las formas más avanzadas: anemia y hemorragias.

Naturaleza de la enfermedad. Ha habido muchas discusiones al respecto. Primeramente se pensó en un origen neoplásico, pero la duración de la enfermedad y la falta de naturaleza destructiva hicieron rechazar esta teoría.

También se pensó en traumatismos, irritaciones e inflamaciones como factores pre- 
disponentes, pero la diversidad de manifestaciones en el organismo no permiten mantener este criterio.

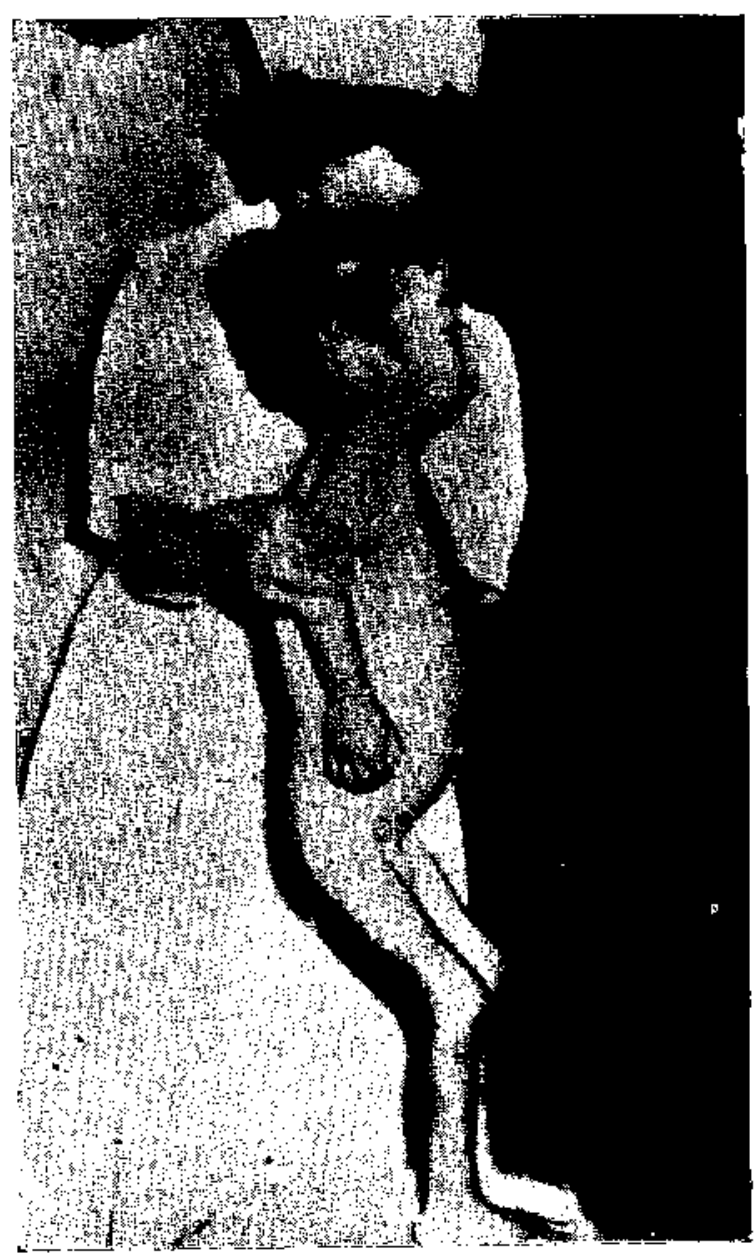

Girifico No 4

Se pensó también en una disfunción pituitaria, teniendo en cuenta la presencia de la diabetes insipida, pero la falta de lesiones microscópicas en esta guándula, en muchos cásos, hizo desechar esta teoría.

La causa familiar y hereditaria parece ser la más importante de las conocidas hasta hoy.

Estudio histológico. En las etapas precoces la biopsia revela el aspecto típico de un granuloma con muy pocas células espumosas. En etapas más avanzadas aparecen las células espumosas que son células xantomatosas con inclusiones de colesterol, sus ésteres y grasas neutras.

Sangre. Los Jípidos sanguíneos en gene-. ral no están alterados y el colesterol se mantiene en cifras normales. El hemograma no es típico y hay discreta anemia. El mielograma puede revelar células espumosas.

Diagnóstico diferencial. En las formas atípicas el diagnóstico diferencial debe hacerse con: diabetes insípida, granuloma no lipoídico, cloroma, mielona, tumores malignos primitivos o metaśtásicos; quistes óseos; osteítis fibrosa quística, Letterei-Siwe, etc.

Evolución. Curso crónico: pueden observarse algunas remisiones espontáneás.

Tratamiento. Ninguno eficaz. Padioterapia; tratamiento endocrino, etc.

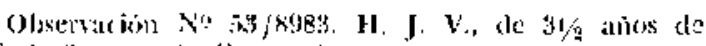
colad. Sevo nuastalino.

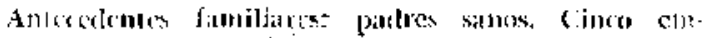

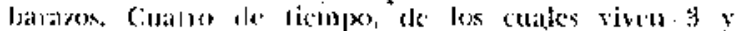

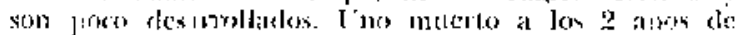

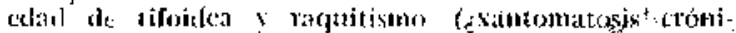
(ai) ; del cual wo heenos prodido recoger antecalenIes. lin abonter. Fay intecelentes de combisencia dia. rante lis dias con in rubercolose grate.

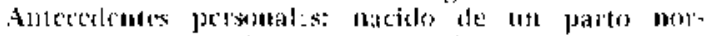

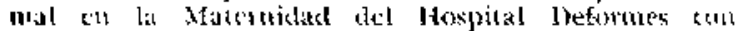

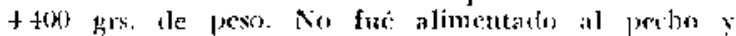
su aldmentación consistió en lache de saca. clirigida en a boliclituce del Ilospitat de Niños de Valpu-

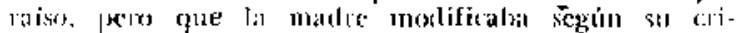

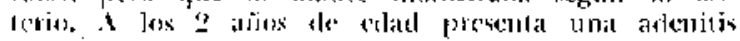

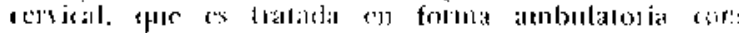

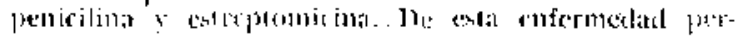
sisten aún manifewationes en el culbo.

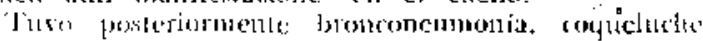

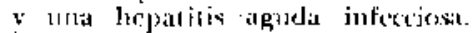

Informe secial: familia yo: vise lumestamente rom

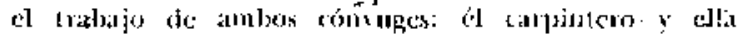

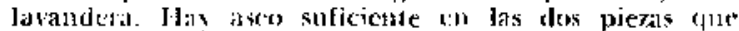

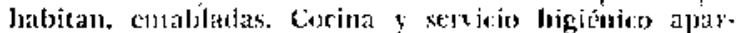

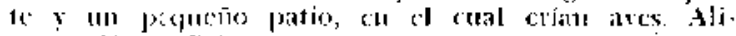
incontacion sufjeiente.

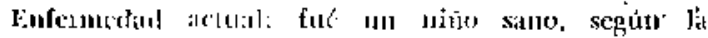
matlre, hasta cl alion :s mests, fecha cll que comell-

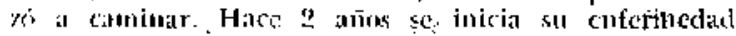

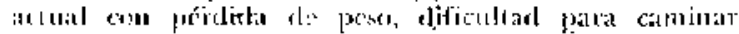

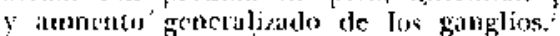

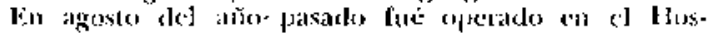
prital de mifios por una atclenitis del: cucllos.

Dessle el comienzo de csta enfermedad, hace ? alios, la madre consulto on repetidas ocasiones por las molestias ale sll hijo, el cual tue semetiklo a va-

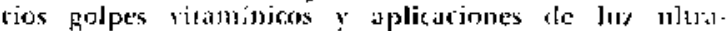

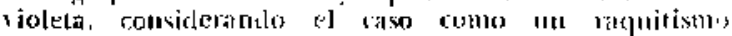
grase. l:l niño liabia perditro la facultatel de cami. nar, sue estater geme:1al (lenbejoraba dia it día.

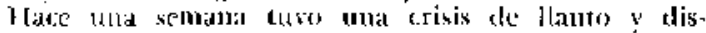
nea, fobril, mux decaids o inopetente. constipilción.

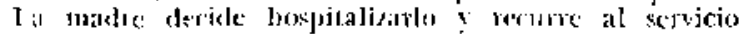

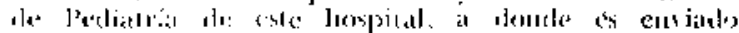

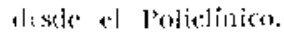

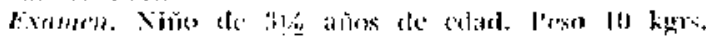

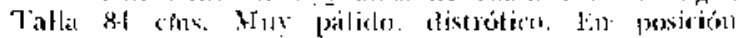

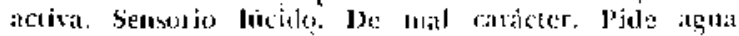
froctemlement:

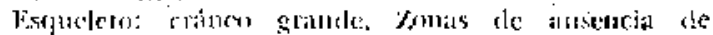
hueses en diferenter isgiones reel crincen! to dife- 


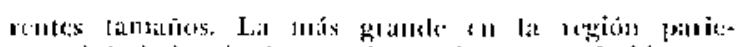

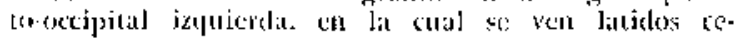
refriales.

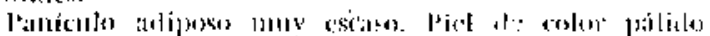

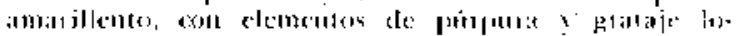

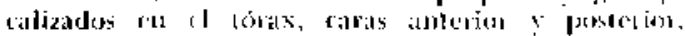

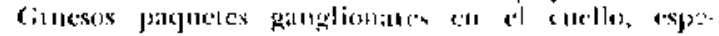

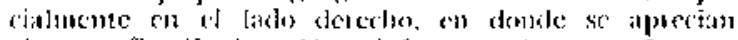

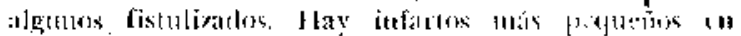
l.1s regionles axilares o iognilizls's

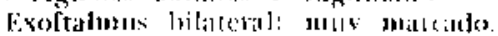

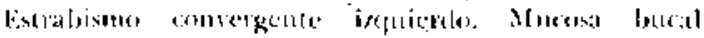

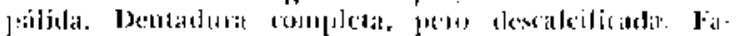
ringe roja colis crutiarlo.

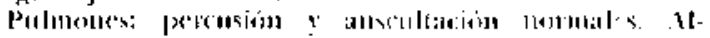

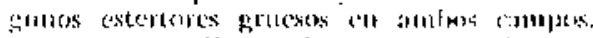

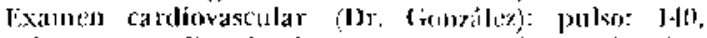

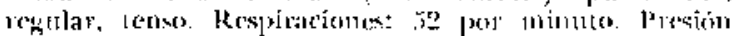

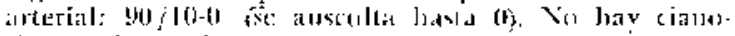
sir $1 \mathrm{i}$ lipoctacismo.

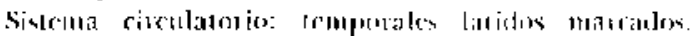

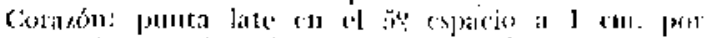

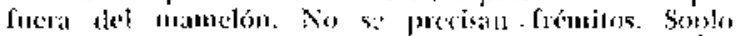

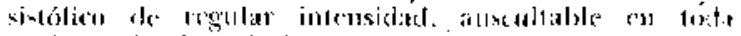

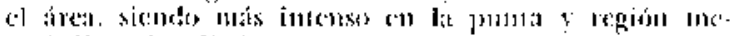

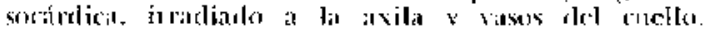

Electrocardiograma: tacmicarrita simlxal. Coranon

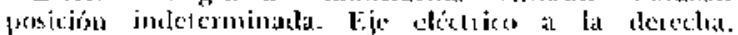

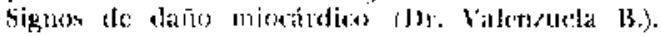

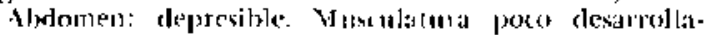

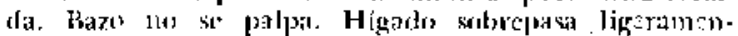

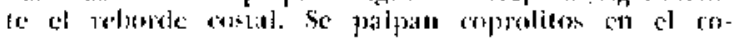
lerth.

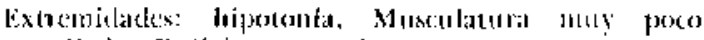
desaboliado. Retlejos notimales.

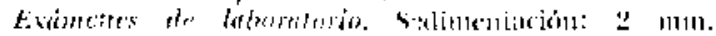

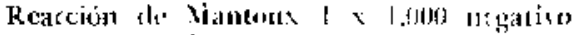

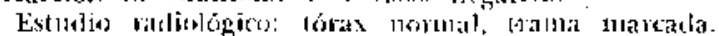

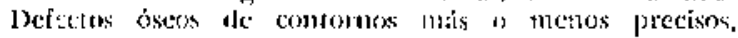

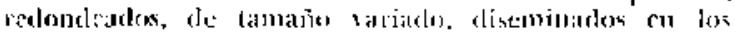

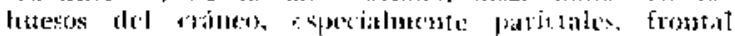

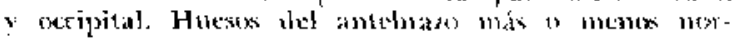

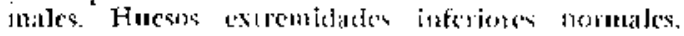

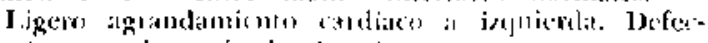

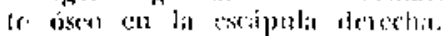

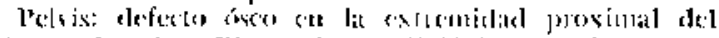

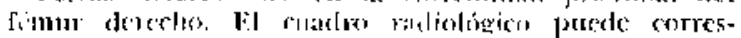

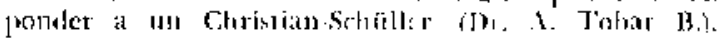

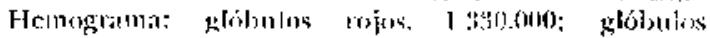

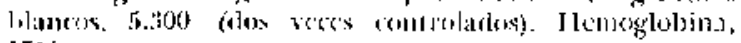
17 :

Mieloritos, 11; jucmiles, 0: bacilifurncs, is: segunen-

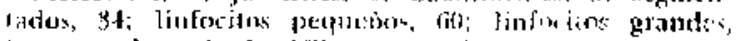
(1) munorites. I: basofites, It: cessingfiles, 0 .

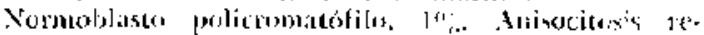

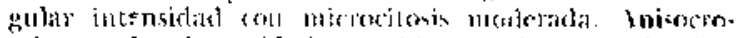

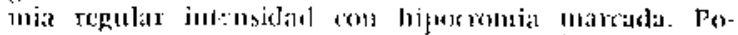

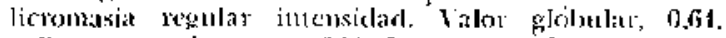

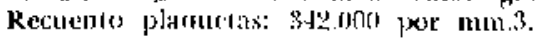

Colesterol: $0.82 \mathrm{gr}$, sion

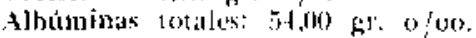

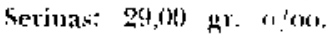

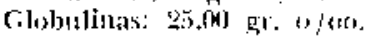

Curciente proteico: 1,1,

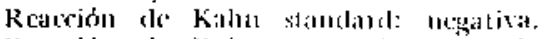

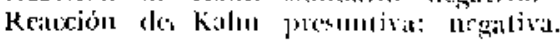

Cilicernia: $[1.90$.

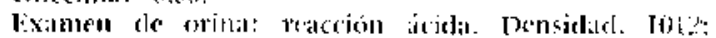

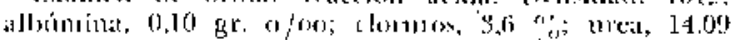

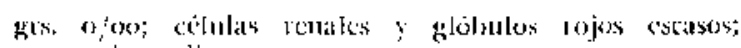
liralo ile sedio escasts.

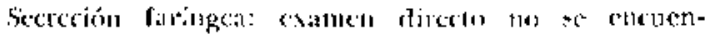

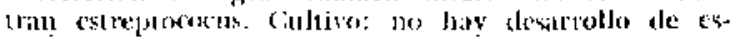

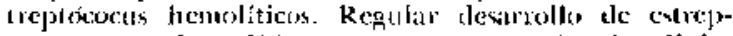

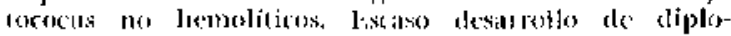
cexus attamilis.

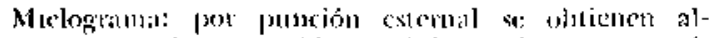
grmas gotals ale comterrido modulits. Al exannell mi-

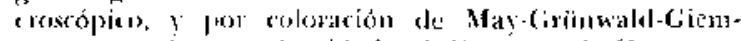

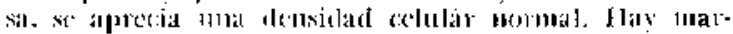

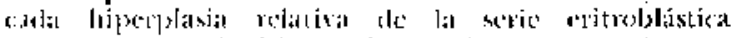

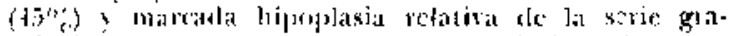

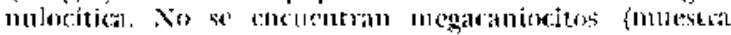

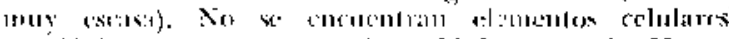

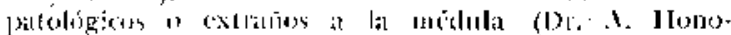
1alo).

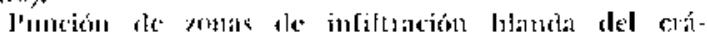
nct: por pumciones repedidas a nivel de las infiltro.

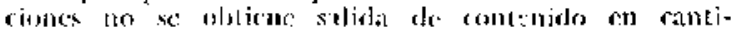

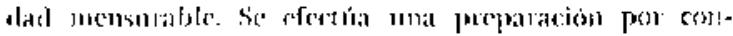

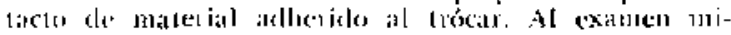

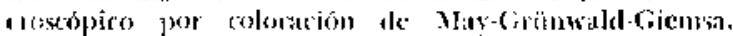

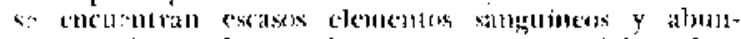

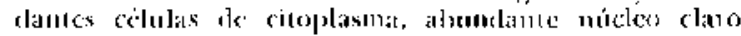

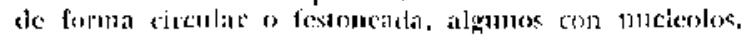

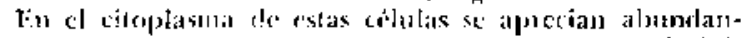
les vacumlas incoloras. I'or sus cardeteres menfoligi-

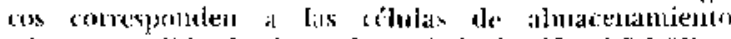
(Sitorage cells) dt' la enfermedial do Hatud-SchüllesChristiall, sic ellotentiall, atetentis, alyumos basófilios

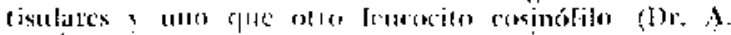
Honosatos).

\section{Comentario}

Se trata de un niño de $3 \mathrm{l} / 2$ años de edad, con antecedentes familiares de alteraciones del esqueleto en dos de sus hermanos, uno de los cuales falleció de tifoidea y raquitismo, según la madre, cuadro que por falta de antecedentes no hemos podido precisar y el otro vivo con síntomas de raquitismo y al cual hasta la fecha no hemos podido examinar. El primero podría haber correspondido a un Hand-Schüller-Christian, enfermedad que en su periodo final evoluciona en forma febril.

La enfermedad se inicia con alteraciones ganglionares y dificultad para la marcha, lo cual es interpretado como un raquitismo y evoluciona en fornta lenta, pero continuada. hasta llegar a nuestro servicio con la tríada típica sintomática de la enfermedad de Hand-Schüller-Christian. Las lesiones de los huesos del cráneo son tan típicas como las descritas en los textos de Pediatría, dando lugar al cúneo geográfico característico en las placas radiológicas. El exoftalmus, nuy manifiesto y la diabetes insipida que se puede apreciar por el hecho de pedir constantemente líquidos. El colesterol en la sangre no está alterado, lo que se describe 
en estos casos, pues, el aumento de colesterol se aprecia solamente en los tejidos.

Hay una disminución de las albúminas totales en la sangre a expensas de las serinas y manifiesta disminución del cucciente. proteico.

La punción de las zonas de infiltración blanda del cráneo demuestran la existencia de células de almacenamiento con las inclusiones características de lipoides.

El examen cardiovascular indica miocarditis con lipoidosis deI miocardio.

Con las pruebas enunciadas podemos decir que pocas veces es posible encontrar un caso de Hand-Schilller-Christian con su sintomatologia $\tan$ completa.

L sbemos hacer presente que por nitransigencia de los padres, solamente nos fué posible tener hospitalizado 3 días a este enfermito en nuestro servicio, tiempo durante el cual debimos practicar los exámenes corres. pondientes, siéndonos imposible conseguir con la madre retenerlo por más tiempo. Durante estos 3 días pudimos observar la evolución febril de este caso, con decaimiento progresivo, sin la menor reacción a transfusiones y terapia con penicilina $y$ estreptomicina.

El niño fallece 2 días después en su domicilio.

\section{Resumen}

Se presenta la observación clínica de un niño de tres y medio años de edad, con un cuadro típico de enfermedad de Hand-Schuiller-Christian ron su tríada característica: cráneo geográfico, exoftalnus y diabetes insipida. Se confirma el diagnóstico con los exámeres de laboratorio respectivos. Se revisa la literatura pediátrica nacional sobre esta enfermedad y se hace un estudio de las santomatosis crónicas, particularmente de la enfermedad de Hand-Schüller-Christian.

\section{Summary}

A case is reported of a typical Hand-Schüller-Christian disease in a three a half year old child. The characteristic triad of "map" skull, exophthalmus and diabetes insipidus was present. The diagnosis was confirmed by the respective laboratory tests. The author revises the national literature regarding this entity and also make a short study of the chronic xantomatosis with special reference to Hand-Schüller-Christian disease.

\section{BLDLIOCiRAIIA}

DBALA GONI, A. Y ISPINOZA, J-Reticulo entoteliosis lipoidicat. Revista Cluilena de Eedialtia $N$ '? 5. Junio de 1944. Santiago de Chile.

IG.ARTLA, FLORENCIO.-Enfermedad de HandSthüller.Chtistiall. Retículo endoleliosis crimeo hipofisiaria. Anais de Facultad $\mathrm{d} \geqslant$ Malicina de Porto Alegre. 1ase I. Julio a retembic. 1938.

ARMAS, CRUL, MONTERO y DIAZ.-"Enfennedarl

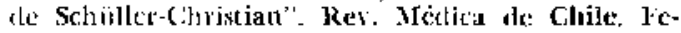

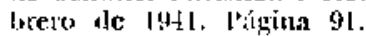

SCROGCilk, A.- "Cirineo geográfico hipolisiario". Alchistes del Hospital Kobsto del Kio. Jubio de 1940. Ṕigina 80.

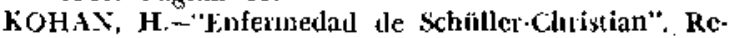
vista Chíkena de Pediatria. 19\$3. Pägina 503.

HAMBIEY E. C.; ARENA, J. M. Y CUYLEV, W. $K$. - "Enfermedad de Hand-Sthtiller.Christian". Anerical Joumal of Isis, of Childien. August 1 tyao.

KARSNER, H. T.-"Sistema reticulo endotelial". Hu. man Patholog!. 60 Edición. 1942.

I.OWE, FRANCIS y FASHEN.A, GLADYS-"Eosimophilic Gramlome of lone". The Jourmal of Pedir. trics. Vol. 3y, 11\}18. Texas.

RREANNEMANN'S.-Practic of Pediatries. Chronic. Idiopathis: Lanthomatoses. V'al. III, chapter 23, píg. 17.

SAILU'N DE: ROIJRIGUE\%, MARIA LIISA-"ICsaturismosis en el nitio". l'ediatria. XIX Curso tle perfeccionamicuto. Aïo I94t6. P'ígina $\$ 03$. Mont+ terijden.

BALZA, J. y CALiBARRLRF, U.-"En caso the ellfermedad de Scliüller-Christian". Socicdad Uruguayil de Pecliatria. Octubre rle 1948.

DFARE RIE'I SHALLAGNE NACH-MANSIFOH."T,as Incjatomegalias policóricas". Sesión 15 de jumin de 39.13. Presse Med. Paris. 43; 8.57, 1935. diull. Soc. Mred, des hop. dc Jaris'.

C.ARRAHAX. ]. I'. Y LASCANO GONZALEL, J. C."Solbre et gramuloma cosinófilo $y$ la enfermedad de Hatul-Schiiller-Cilnjstian". Arch. Arg. de Pediatía. Año XY. T. XXIC. N? 1. Julio de 1944.

WAI,GREN, A-Sisternic. Reticulo Endothelial granulowa. Non. lipoid Reticulo endoheliosis and Schïller-Christian. Discase.

SOSMIN, M. C.-Xantomatosis. Scliültet Cituistian Dis. ]. $\rightarrow$. N. A. $9 x:$ I 10, 1932. 